\title{
Toma de decisiones del gerente de proyectos, a partir del seguimiento de un proyecto de construcción.
}

Resúmen

En la industria de la construcción cada proyecto involucra variables que lo hacen único. A pesar de la planeación, existe un porcentaje de incertidumbre que hace que su manejo deba ser adecuado con las circunstancias y características de cada momento. Recopilar, documentar y generar lecciones aprendidas sobre la experiencia que adquieren los actores involucrados son actividades que permiten desarrollar una herramienta para el gerente de proyectos, quien retomará la información en los momentos necesarios como apoyo en la toma de decisiones. El gerente de proyectos debe promover oportunidades para ampliar conocimiento con base en la experiencia, de tal manera que esta herramienta sea útil para mejorar el desempeño en nuevos proyectos, analizando objetivamente las actividades anteriores para documentar con certeza los procesos exitosos, los factibles de ser corregidos y aquellos que no deben ser repetidos para así evitar errores y repetir aciertos, tomando decisiones cada vez más precisas.

Abstract

In the construction industry every project implicates their own respective variables. Despite planning, there always exists a percentage of uncertainty; making it important that management is carried out in accordance with the circumstances and characteristics of every moment. Collecting, documenting and generating lessons learned from experiences acquired by the actors involved are activities that allow to develop a tool for the management of projects who will take back the information in the necessary moments as support for making decisions. The management of the projects should promote opportunities to expand knowledge with an emphasis in gained experience. In this manner, this tool will be useful to improve the performance of new projects, analysing objectively the activities of past projects so as to document with certainty successful processes, those feasible of being corrected and those that should not be repeated. In this manner, one will avoid errors and to repeat successes, making decisions every time more exact

\author{
Edgar Sánchez \\ esanchez@uniminuto.edu
}

\section{INTRODUCCIÓN}

En la industria de la construcción cada proyecto involucra variables que lo hacen único. A pesar de la planeación, existe un porcentaje de incertidumbre que hace que su manejo deba ser el adecuado con las circunstancias y características de cada momento. Recopilar, documentar y generar lecciones aprendidas, de la experiencia que adquieren los actores involucrados, son actividades que permiten desarrollar una herramienta para el gerente de proyectos, quien retomará la información en los momentos necesarios como apoyo en la toma de decisiones.

El gerente de proyectos debe promover oportunidades para ampliar conocimiento con base en la experiencia, de tal manera que esta herramienta sea útil para mejorar el desempeño en nuevos

proyectos, analizando objetivamente las actividades de proyectos anteriores para documentar con certeza los procesos exitosos, los factibles a ser corregidos y aquellos que no deben ser repetidos para asi evitar errores y repetir aciertos, tomando decisiones cada vez más precisas.

\section{JUSTIFICACIÓN}

La gerencia de proyectos como actividad es novedosa y cada día se hace más importante. Debido a los problemas que se han presentado en el desarrollo de los proyectos se impone como una solución bastante atractiva, pues permite realizar coordinación de actividades, enfocar los objetivos en tiempo, costo y calidad acorde con las necesidades del cliente.

Dada la naturaleza tan variada de los diversos proyectos en la ingeniería, el gerente debe estar presente a lo largo de las etapas velando por el buen desarrollo de éstas, llevando en armonía a todos los participantes con la planeación elaborada para lograr los objetivos propuestos.

La toma de decisiones adquiere un gran significado en la labor del gerente de proyectos y debe indicar la dirección correcta que se debe seguir. Una gran experiencia es garantía en esta labor, pero en ocasiones se puede fallar. 
Al documentar cuidadosamente los procesos sobre los cuales se adquiere experiencia y luego realizar un análisis íntegro, identificando aciertos y errores, se logra una herramienta adicional que será un valioso apoyo de referencia en los momentos en que se deban tomar éstas decisiones o, simplemente, podrán ayudar a guiar actividades con base en las ya realizadas.

Dadas las características particulares que envuelven los proyectos de construcción es importante realizar su seguimiento en su aspecto gerencial.

El seguimiento realizado al proyecto edificio Mario Laserna comprende el registro de la información extraída del proceso gerencial desarrollado en los aspectos legal, administrativo, económico y financiero, comercial y técnico.

La Universidad de los Andes en su compromiso de dar respuesta desde el terreno educativo a las necesidades reales del país y aportar a la evolución del mismo ha consolidado una serie de políticas encaminadas a cumplirle a la sociedad colombiana y mundial.

Para lograr tal fin, desarrolla y pone en práctica metodologías de avanzada en la docencia y la investigación, orientadas a que el estudiante sea el principal agente de su formación y resuelva los problemas que se le presenten con creatividad y responsabilidad.

El proyecto edifico Mario Laserna hace parte del compromiso de construir infraestructura que compense la gran necesidad de involucrar un mayor espacio de experimentación dentro de las actividades curriculares.

Actualmente, son pocas las universidades que poseen centros de desarrollo de aprendizaje e investigación como el proyecto edificio Mario Laserna que se está construyendo en la Universidad de los Andes, que surgió de varias ideas que fueron propiciadas como consecuencia de diversas visitas hechas por un grupo de profesores de la facultad de ingeniería cuando regresaron de conocer la experiencia acumulada de pares internacionales en las universidades del grupo de pioneras a nivel mundial, en el tema de investigación.

El desarrollo de este proceso compromete un apoyo de tipo conceptual a fin de poder lograr una mayor y mejor ubicación en el contexto. La idea fundamental es determinar con claridad un conjunto de conocimientos, que permita dirigir el proceso elaborado en la forma más adecuada y con la mejor referencia a los términos que se utilicen. Es importante tener la idea global e ir descomponiéndola en ideas particulares que muestren las necesidades propias, de manera que se logren concatenar entre ellas.

Un proyecto se genera con el propósito de satisfacer una necesidad, solucionar un problema o generar una oportunidad de inversión. Está dentro de un marco limitante de recursos que se maneja mediante un presupuesto, plazo que se maneja mediante una programación y unas especificaciones de calidad.

La gerencia es una disciplina novedosa en el medio, pero se hace necesaria en todo proyecto, de manera que permita generar coherencia en los objetivos de costo, tiempo y calidad, manejar la "batuta" utilizando clara comunicación, realizar seguimiento y retroalimentación oportunos.'

La gerencia de proyectos es una labor que abarca actividades de tipo administrativo, legal, financiero y económico, técnico y comercial, con el fin de realizar la gestión para la iniciación y desarrollo de un proyecto inmobiliario. ${ }^{2}$

La descripción de estas funciones otorgadas al gerente de proyectos se encuentra en el Decreto 2090 de 1989 y es la única reglamentación existente elaborada por la Sociedad Colombiana de Arquitectos.

La labor desarrollada por la gerencia de proyectos es fundamental debido a la naturaleza de éstos. Un proyecto es único e irrepetible, cuenta un inicio y un fin de tal manera que está encerrado en un tiempo, lo que lo hace temporal. Es complejo debido a que es multidisciplinario, maneja niveles de incertidumbre que afectan los parámetros de costo y tiempo, pero que van reduciéndose con el transcurrir de su avance y también está afectado por un entorno.

La toma de decisiones y el esfuerzo para realizar cambios genera impacto en el proyecto. La mejor manera para evitar esta consecuencia es una buena planeación inicial, en la que el gerente de proyecto juega un papel muy importante. 


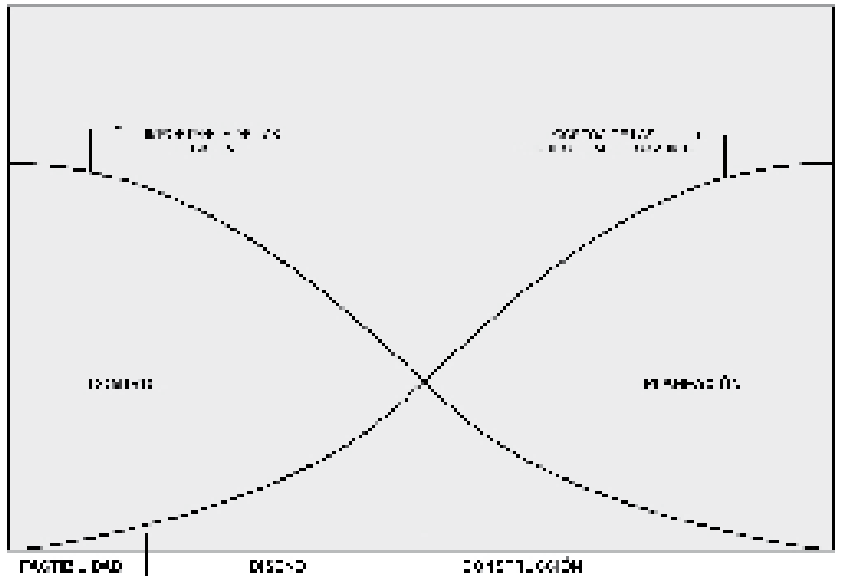

Figura 1. Importancia de las decisiones y esfuerzo para hacer cambios y correciones. ${ }^{3}$

Teniendo en cuenta las fases del desarrollo de un proyecto como son: factibilidad, diseño, construcción y puesta en marcha, se podría afirmar que la mayor incertidumbre está generada en la etapa de factibilidad, la cual va disminuyendo con el transcurso de su desarrollo y en la medida en que se avanza en las demás etapas.

La gerencia de proyectos exige desarrollar mecanismos para llevar a cabo el control de los procesos y su ajuste a la planeación organizada en un inicio. Este mecanismo debe permitir la visualización de los procesos que no estén bien encauzados, que generen desviaciones en tiempo, recursos o calidad, de tal manera que se convierta en una herramienta que aporte claridad y que permita la toma de decisiones a fin de corregir dichas desviaciones.

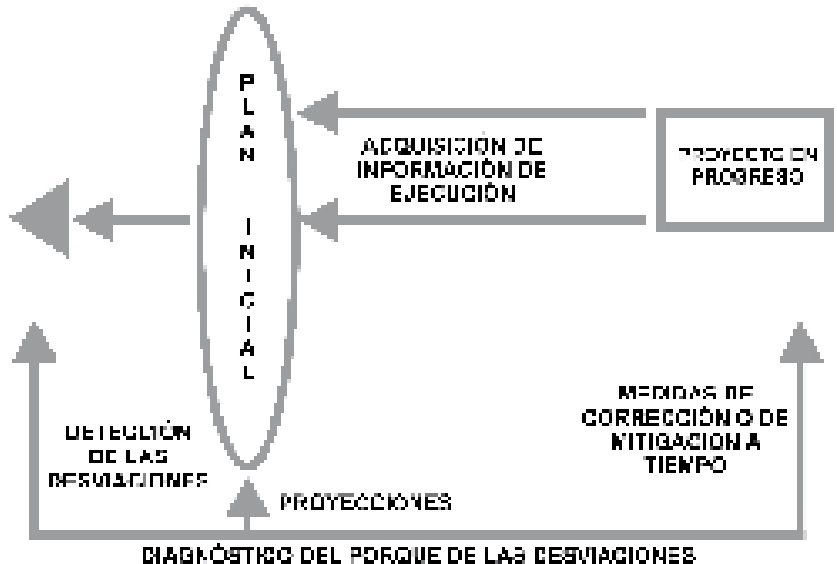

CHAGROSTKC DEL PDROUE DE LAO CESIIACIONES

Figura 2. Ciclo de control de un proyecto. Gerencia de proyectos, Diegi Echeverry, 2005.

\section{El manejo ambiental}

El manejo ambiental es un aspecto fundamental que permite generar conciencia sobre el cumplimiento de las normas establecidas en la legislación para la ejecución de un proyecto, además permite avanzar en el desarrollo de conductas que se amolden a una filosofía de mejoramiento del impacto que se pueda acarrear tanto en el entorno, como en su interior.

\section{La seguridad industrial}

Es importante hacer una reflexión acerca del tema y realizar un acercamiento real a su normatividad y legislación. En Colombia son evidentes las deficiencias en materia de salud y seguridad causantes de un gran número de lesiones, muertes y deterioro en las condiciones del trabajador de la construcción.

Llevar a cabo un seguimiento de la accidentalidad en los proyectos es base fundamental para hacer un análisis que permita corregir las deficiencias existentes y reducir sus implicaciones, en algunos casos trágicas, que dejan huellas y afectan de manera considerable los proyectos.

Desarrollar un programa de salud ocupacional teniendo en cuenta la particularidad de cada proyecto, que contemple higiene y seguridad industrial para la identificación y control de riesgos, involucrando a todos los actores, realizando una capacitación en busca de un cambio de conciencia dirigida hacia la seguridad es una herramienta que generará beneficios.

Adicionalmente, generar y difundir un programa de atención de emergencias y desastres que tenga una brigada que esté como responsable y coordinadora hará parte complementaria al programa de salud ocupacional.

\section{La gestión del riesgo}

Se puede definir el riesgo como la posibilidad que existe en un proyecto de ser afectado por un elemento, en donde dicha afectación puede causar un daño, de manera que altere su desarrollo normal.

La gestión del riesgo es una labor indispensable en la gerencia, no es desconocido que grandes proyectos han fracasado debido a 
la mala visión y el manejo dado a la exposición de riesgos.

Existen factores que deben tenerse en cuenta y que podrían minimizar los riesgos como: manejar las presiones ejercidas al proyecto, el adecuado uso de la nueva tecnología, el buen desempeño en los procedimientos de operación, la claridad acerca de roles y responsabilidades, la adecuada planeación, la incertidumbre que se genera al comenzar un proyecto y que se mantiene en nivel de descenso acorde con su avance, los factores externos, la toma de decisiones adecuada, en fin son un número muy variado de factores que pueden en determinados momentos generar éste problema.

Las fuentes de riesgo más comunes en un proyecto son: contractuales, técnicas, financieras y externas. Los riesgos contractuales hacen mención a los deberes, derechos y responsabilidades de las partes en un contrato, la falta de claridad y entendimiento y la mala administración e implementación de éste.

Los riesgos técnicos están relacionados con la incertidumbre tecnológica. Los riesgos financieros están relacionados con malos estimativos en las propuestas, la disponibilidad de recursos y la eficiencia en el manejo de éstos.

Los riegos externos tienen que ver con cambios en los requerimientos y decisiones del cliente y los cambios en la legislación.

La etapa inicial en el desarrollo del proceso de manejo del riesgo es la identificación de éste, cabe añadir que un riesgo no identificado no puede ser manejado. La identificación demanda habilidad, experiencia y criterio de todo el personal del proyecto.

Transcurrida la etapa de identificación, el riesgo debe ser cuantificado en cuanto al impacto que generará en todos los aspectos, de esta manera es posible afirmar que el riesgo está dado en función de la incertidumbre y del impacto generado:

\section{Riesgo (Incertidumbre, Impacto) ${ }^{4}$}

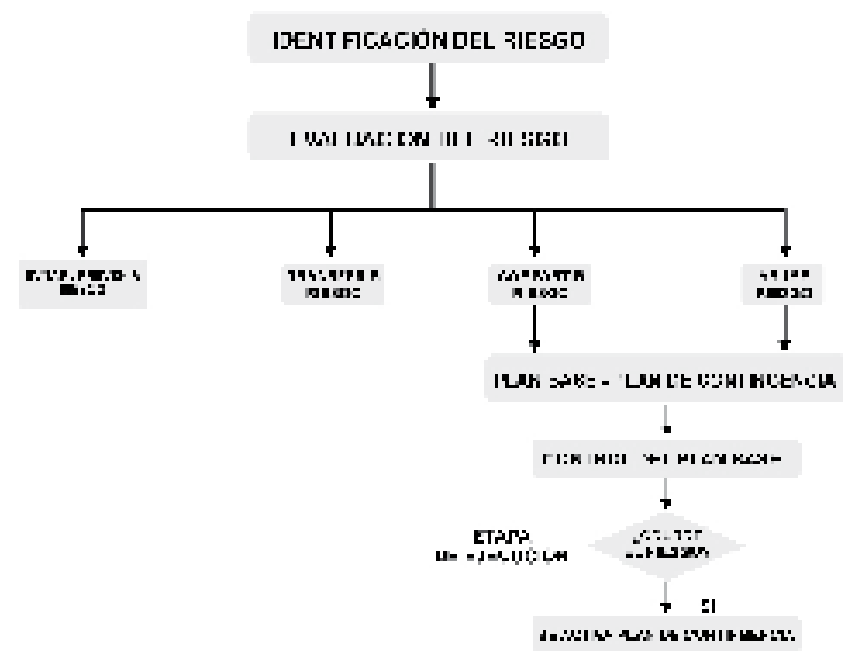

Figura3. Administración de riesgo Gerencia de proyectos, Diego Echeverry, 2005.

\section{El aspecto técnico}

Los aspectos técnicos desplegados en el desarrollo de la fase constructiva de un proyecto poseen una fundamentación conceptual que permite analizar su desempeño y ver, técnicamente, los pasos de cada proceso.

Por esta razón se citan conceptos básicos acerca de dichos procedimientos en las etapas de excavación, cimentación y estructura parcial, a fin de ofrecer una base sólida para el lector.

La metodología que fue usada en el desarrollo de este trabajo fue la de registro histórico del proceso gerencial del edificio Mario Laserna en sus aspectos legal, administrativo, técnico, comercial y financiero mediante documentación periódica en las etapas de diseños y construcción parcial, por medio de seguimiento en obra, entrevistas a personas vinculadas directa e indirectamente al proyecto, entrevistas a personas que conocen acerca del tema, registro fotográfico y fílmico de procesos técnicos, clasificación y organización del material elaborado, de forma que se desarrolle un enriquecimiento al proceso de tesis. Adicionalmente, existió apoyo de consulta bibliográfica, búsqueda de información en Internet y consulta en la información adquirida en los cursos de maestría.

A medida que se recolectaba esta información se realizó un análisis sobre enseñanzas generadas por el proceso gerencial, de forma que se mencionan lecciones aprendidas a considerar 
en proyectos futuros como aquellas que se consideran importantes por su gran éxito e impacto, aquellas que dejan los procesos que están sujetos a mejoras y las que ofrecen los procesos que se considera no se deben repetir.

Este proceso de seguimiento se inició en el mes de noviembre del año 2004 y culmina con la entrega del documento de esta tesis. La labor se programó de la siguiente manera:

- Documentación previa sobre el proyecto.

- Consecución de permisos en la universidad para acceder al proyecto.

- Ingreso al proyecto.

- Programación semanal de actividades como visitas de obra, entrevistas con el personal involucrado en el desarrollo, seguimiento a comités de obra y actividades desarrolladas al interior del proyecto.

- Asistencia a eventos como conferencias, charlas y reuniones relacionadas con el proyecto edificio Mario Laserna.

- Recolección de información, registro fotográfico y filmico.

- Investigación sobre documentos como artículos, tesis de maestría y publicaciones acerca del proyecto.

Todas las actividades mencionadas se desarrollaron ininterrumpidamente durante el año de seguimiento lo que permitió una efectiva interacción con todo lo relacionado al proyecto.

\section{Objetivos del proyecto edificio Mario Laserna}

Teniendo en cuenta la misión de la Universidad de los Andes que busca la excelencia académica e imparte en sus estudiantes una formación crítica que afianza en ellos la conciencia de sus responsabilidades sociales y cívicas, así como su compromiso con el análisis y solución de los problemas del país, el proyecto edificio Mario Laserna hace parte de un grupo de obras realizadas por la universidad con el fin de brindar mejores herramientas en busca de cumplir el compromiso hacia la sociedad y el país. ${ }^{5}$

Enmarcando el proyecto dentro del desarrollo de infraestructura de la Universidad existen objetivos como:

- Albergar el desarrollo de la Facultad de Ingeniería en todos sus programas actuales y los programas que estén proyectados a desarrollar en sus actividades académicas, administrativas y de investigación.

- Buscar un diseño integrado para el manejo de los recursos, haciendo uso del concepto de auto sostenibilidad y manteniendo buena calidad en su ambiente interior.

- Aportar un espacio adecuado para el desarrollo de la biblioteca Ramón de Zubiría.

- Garantizar una adecuada articulación con la infraestructura actual de la Universidad.

- Ser un edificio con espacios que permitan generar comunidad.

- Permitir la interacción K12, es decir la interacción de estudiantes desde el nivel de kinder hasta un nivel máximo de secundaria, para asi promover desde el colegio una cercanía hacia la ciencia y la tecnología.

\section{Descripción general del proyecto edificio Mario Laserna}

El proyecto edificio Mario Laserna tiene proyectado en su interior 3 sótanos y 8 niveles de piso, dentro de los cuales existen laboratorios, zona de biblioteca, la calle del saber, un auditorio en dos niveles, aulas, oficinas para profesores, salas de reunión, zona de parqueo y zona para labores administrativas.

Contará con un puente peatonal que servirá como comunicación con la zona del edificio actual de Ingeniería, el cual será demolido tan pronto se termine el proyecto edificio Mario Laserna.

\section{Aspecto Gerencial del Proyecto Edificio Mario Laserna}

El desarrollo de un proyecto de construcción implica para el gerente de proyectos esfuerzos en varios aspectos, bajo estos parámetros se describe el proceso gerencial del proyecto edificio Mario Laserna.

\section{Aspecto legal}

El gerente de proyectos debe contribuir en la dirección de la gestión legal ante los respectivos entes, a fin de cumplir con todos los requerimientos para obtener los permisos necesarios para desarrollar el proyecto.

En este aspecto se relacionan actividades como la licencia de construcción y permisos adicionales obtenidos con el objetivo de iniciar el proyecto.

5. Seguimiento del desarrollo del proyecto del nuevo edificio Mario Laserna de ingeniería de la universidad de los Andes, Alex Wihiler 
Es importante mencionar la participación en el proceso de obtención de estos permisos del Ministerio de Cultura, la Corporación la Candelaria, el Departamento de Planeación Distrital y la dirección de Planta Física de la Universidad de los Andes.

\section{Aspecto Técnico}

El gerente de proyectos define claramente los objetivos en costo, tiempo y calidad del proyecto. Para cumplir con estos objetivos se deben aprobar los diseños, especificaciones y plazos de ejecución, además de supervisar los procesos constructivos. ${ }^{6}$

En el análisis de seguimiento es importante recopilar la información de estos procesos, para el caso particular del proyecto edificio Mario Laserna se desarrolló un marco conceptual acerca de los procesos llevados a cabo dentro del tiempo de ésta investigación, se documentó y recopilo información, y se realizó un registro fotográfico sobre los procesos ejecutados.

Los procesos incluidos en el seguimiento son: excavación, armada y fundida de caissons, muros pantalla, anclajes, cimentación y estructura parcial, de manera que se cuente con la referencia en diseño y metodología utilizada en el desarrollo de estas actividades.

Un aporte que parte del seguimiento en particular del proyecto edificio Mario Laserna realizado para tener en cuenta en otros procesos de seguimiento es un esquema en forma de diagrama de flujo del desarrollo de los procesos constructivos, que permite establecer los pasos y su manejo en particular. Es importante que cada uno de los pasos mencionados en el proceso esté descrito, tenga registro fotográfico y fílmico para lograr un completo registro histórico, de manera tal que permita hacer un análisis comparativo con procesos similares.

Ver Figura 4

\section{Aspecto Administrativo}

Definir el organigrama bajo el cual debe operar el proyecto, políticas para el manejo de riesgos, definir procedimientos para la apertura de licitaciones y el manejo de contratación, son labores de la gerencia de proyectos.

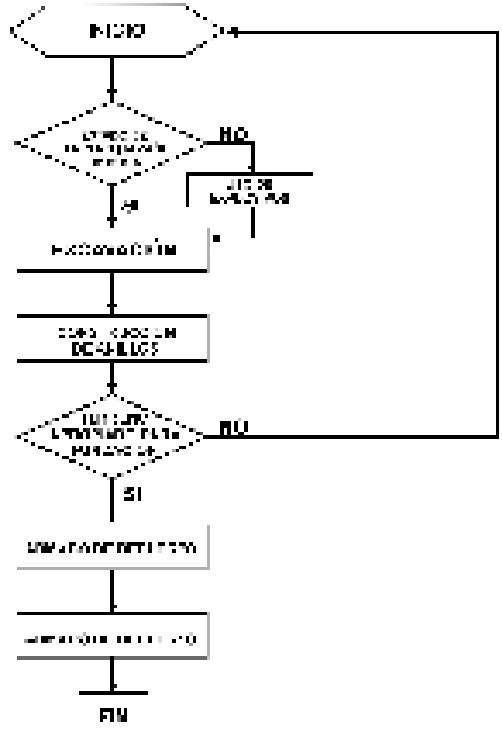

Figura 4. Proceso de construcción del caisson

El manejo dado en contratación a los costos indirectos se considera particular y se describe a continuación:

A (Costos de administración).

- Inscrito en un formato generado para éste propósito.

- No es porcentaje sobre el valor de costos directos.

- Es un valor fijo mensual debidamente sustentado en el formato.

- En caso de que la actividad contratada excediera el tiempo establecido y debiera ser reprogramada, el primer mes de administración no se pagaría y de ser mayor el tiempo para terminarla, el valor cancelado se ajustaría al previamente establecido y justificado.

I + U (Imprevistos + Utilidad)

- Si los costos directos por obras adicionales o mayores cantidades se incrementan en un \% mayor o igual al $15 \%$ de los costos directos iniciales, el reconocimiento es proporcional al $\mathrm{l}+\mathrm{U}$ inicial.

En caso de no superar el 15\% el l+U no se incrementa.

\section{Aspecto Comercial}

El proyecto edificio Mario Laserna está enfocado en el aspecto netamente académico y en particular a ser una edificación que proporcione herramientas a nivel de investigación y experimentación, por tanto no está programado para ser vendido, lo cual excluye el análisis en el aspecto comercial.

6. Decreto 2090, Sociedad Colombiana de Arquitectos, 1989 


\section{Aspecto Financiero}

El manejo financiero del proyecto edificio Mario Laserna está directamente relacionado con la Universidad de los Andes y proviene de recursos propios de la Universidad.

\section{Gerencia y Toma de Decisiones}

El gerente de proyectos debe tomar decisiones adecuadas en momentos precisos, por esta razón acorde con la situación en determinados momentos existió esta necesidad en el proyecto edificio Mario Laserna, lo que conllevó a determinar decisiones en aspectos como: el número de caissons, cambio de muros anclados, manejo de altura en el edificio, cambios arquitectónicos y losa de entrepiso.

\section{Reducción en el número de caissons}

Una decisión importante en su momento fue un cambio en la cimentación debido al cambio en el número inicial de caissons proyectado para el edificio Mario Laserna.

Es importante tener en cuenta que el impacto en el aspecto económico debido a ésta reducción fue grande, de manera que se logró seguir cumpliendo el objetivo y reducir costos.

\section{Cambio en muros anclados}

Cuando se desarrolla una actividad, existen situaciones que pueden alterar su programación, es el caso del diseño de muros pantalla y anclajes elaborado para contener los esfuerzos generados por la excavación en los taludes laterales.

Inicialmente, estaba planeada la construcción de estos muros en todo el contorno; pero, surgieron dos situaciones que hicieron tomar decisiones que alteraron ésta planeación.

La primera surgió cuando se desarrollaba la excavación y se encontró sepultada la antigua construcción que perteneció en su entonces a la cervecería Germania. Particularmente, ésta construcción contenía un muro al cual se le hizo un análisis de estabilidad y se determinó que podía cumplir la función de los muros pantalla proyectados para la zona en la cual estaba ubicado.

La segunda se presentó sobre el costado de la Calle $19^{a}$ y tuvo que ver con una canalización del río San Francisco. Dicha canalización pasa bajo la calle y utiliza un tubo cuya localización no se tenía con exactitud, razón por la cual se determinó no realizar anclajes a los muros pantalla de este sector y en su reemplazo se utilizaron muros de contrafuerte que permitieron hacer las veces de los anclajes para sostenerlos.

\section{Afectaciones a los vecinos}

Por lo general, el proceso constructivo de una edificación genera consecuencias en los terrenos colindantes, de tal manera que dichas consecuencias deben ser corregidas a fin de subsanar los inconvenientes que se puedan ocasionar.

La ley señala la responsabilidad que tiene el constructor sobre los riesgos que se corre al realizar una obra, al igual que el código civil define la responsabilidad que tiene el propietario sobre el daño que se cause en una edificación vecina extendiéndola a los encargados de realizar la construcción.

La gerencia del proyecto en unión con la Universidad de los Andes generó una política de manejo acerca del tema con los vecinos que pudiesen ser afectados por el proyecto que se inició con la constitución de actas de vecindad previas al inicio de los trabajos de construcción y en conjunto con cada vecino del predio, en donde se dejó constancia de la valoración del estado de cada edificación.

Adicionalmente, durante el transcurso del proceso constructivo del edificio Mario Laserna, la gerencia del proyecto ha estado muy pendiente de los problemas que han presentado las edificaciones vecinas, corrigiéndolas a la mayor brevedad de manera que exista un excelente ambiente de cordialidad y satisfacción en las relaciones entre las partes.

\section{Manejo de altura}

Durante el proceso de obtención de la licencia de construcción, se estableció una altura bajo la cual se debería desarrollar el proyecto edificio Mario Laserna. El desarrollo del proyecto arquitectónico tuvo un punto de referencia equivocado y se constituyó un sobrepaso en la altura total determinada para el edificio. Al notar el desfase de altura fue importante determinar la solución para hacer la corrección.

La decisión para corregir el sobrepaso fue rediseñar los elementos estructurales que afectan directamente el edificio en el tema de altura y así lograr adecuar nuevamente el proyecto a la altura inicialmente planeada. 


\section{Cambios arquitectónicos}

Aún cuando existe un plan inicial, generado con base en el desarrollo de estudios y diseños aprobados por los actores involucrados, existen cambios constantes en varios aspectos, caso particular el aspecto arquitectónico que se ha visto afectado en su desarrollo, con las consecuencias que ello implica en otros aspectos como por ejemplo el estructural.

\section{Lecciones Aprendidas y Recomen- daciones}

La experiencia adquirida al realizar el seguimiento del proyecto edificio Mario Laserna deja un valor agregado al hacer abstracción del análisis de los procesos y se refleja en los siguientes aportes:

\section{Seguridad industrial}

Como se mencionó en un comienzo, la importancia que está tomando el tema de la seguridad industrial es muy válida, dadas las experiencias que se han presentado en proyectos anteriores y las consecuencias que han existido.

Como aspecto a tener en cuenta, es de gran aporte guiar la industria de la construcción en la dirección de implementar de forma obligatoria un plan que contenga la toma de medidas a fin de tener un control, mantener políticas de manejo e implementar procesos acerca del tema de la seguridad industrial.

La gerencia de proyectos debe involucrar dentro de sus labores el manejo de estas actividades y velar por su cumplimiento por parte de los actores del proyecto.

\section{Cultura del obrero ante el riesgo}

El comportamiento asumido por el obrero ante situaciones que ponen en riesgo su integridad personal no es en ocasiones el mejor, se desconocen las normas básicas de seguridad o simplemente a pesar de conocerlas se hace caso omiso de ellas.

Esta situación, agrega un alto porcentaje de riesgo que no debería asumirse si existiera la conciencia en el obrero de las consecuencias a las que se está expuesto debido a su comportamiento.

El avance en la industria de la construcción en comparación con otras industrias ha sido real- mente muy bajo, si a esto se suma que la capacitación recibida por el obrero también lo ha sido, bien sea por falta de interés o por no contar con la capacidad económica para hacerlo, esto hace que la industria en su nivel de ejecución se desenvuelva dentro de una cultura en la que no se da la importancia indispensable a las normas y éstas se consideran a veces innecesarias, puesto que la experiencia adquirida, quizás la suerte o la falta de visión a consecuencias futuras ha hecho que se sigan repitiendo errores.

\section{Concertación de diseños}

En la planificación de un proyecto de construcción existen diversas áreas que se deben integrar para generar el mejor plan que ocasione el menor impacto negativo y que proporcione los mejores resultados en costo, tiempo y calidad.

El gerente del proyecto debe involucrarse y ser pieza fundamental en el acople de las ideas propuestas por cada una de los actores, siempre llevando la "batuta" con liderazgo, manejo global del conocimiento, habilidad en negociaciones, manejo de conflictos, una buena teoría para tomar decisiones y optimización de procesos.

Desarrollar una excelente planeación con objetivos claros para el proyecto es una base de éxito, pero si por el contrario, la planeación no es buena, existe un alto riesgo de incurrir en constantes cambios durante el desarrollo de la etapa constructiva del proyecto.

En la Figura 1, se establece con claridad el concepto de impacto que generan los cambios durante las etapas del proyecto, siendo más traumático y perjudicial realizarlos en la medida en que el proyecto tenga un mayor avance.

Cada proyecto es único, de manera que las circunstancias que lo envuelven son propias.

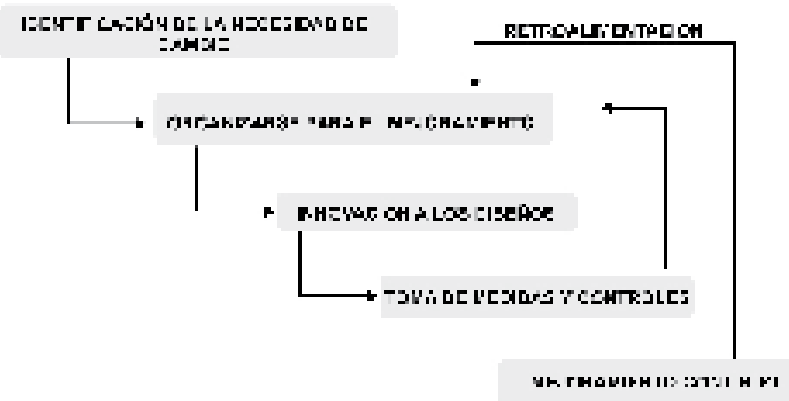

Figura 5. Cambio para el mejoramiento 


\section{Programación}

Los tiempos estimados en el caso particular del proyecto edificio Mario Laserna en la programación de actividades han sido reprogramados en varias ocasiones debido a factores como bajo rendimiento, porcentaje mayor al estimado en cantidad de roca en el proceso de excavación y cambios en diseños.

Debido a ésta situación es posible afirmar que existen factores externos que pueden afectar un proyecto, como los mencionados en el caso específico del edificio Mario Laserna y que no son fácilmente previsibles, estando contemplados dentro de los riesgos que se corren en la ejecución de todo proyecto.

Una excelente planeación puede ser totalmente alterada debido a situaciones que no son fácilmente predecibles y que no estén contempladas en ella. Sin embargo, existen otras situaciones que es importante considerar debido al gran impacto que pueden generar en un proyecto y que son previsibles desde su comienzo.

Los cambios constantes que el cliente solicita para ajustar las necesidades que se presentan a lo largo del desarrollo del proyecto afectan notoriamente la organización desarrollada y orientada bajo una planeación existente, lo que causa la realización de esfuerzos no esperados y otras veces innecesarios, generando desgaste en el equipo de trabajo.

La labor del gerente debe estar orientada a reducir el riesgo del impacto en recursos, programa y alcance. La administración del cambio demanda mantener un equilibrio en estos aspectos.

\section{Presupuesto}

Generalmente, se ajusta a los proyectos con gran exactitud una frase muy contundente "el mejor presupuesto de un proyecto siempre es el último", pero en la medida en que se realice un buen trabajo de planeación, teniendo en cuenta todas las posibles variables, se podrá lograr al inicio un estimativo muy cercano al valor real del proyecto.

\section{Oferta mercantil}

Dada la importancia en el manejo de contratación en suministro de insumos, la oferta mercantil surgió como un elemento que permitía bajo el concepto claro y a la luz jurídica evitar el pago del impuesto de timbre.

La oferta mercantil está gravada con el impuesto de timbre, sólo cuando es aceptada por el destinatario, momento en el cual se perfecciona un contrato, salvo que se acepte mediante una orden de compra, razón por la cual la misma no se debe asimilar a un contrato.

Para evitar incurrir en el riesgo de no cumplir en el ámbito tributario es conveniente al considerar documentos como ofertas mercantiles, tener en cuenta que no incluyan elementos que expresen manifestación bilateral de voluntades, es decir que a todas luces se manifieste participación de la voluntad de dos partes, al incluir cláusulas que hagan mención al respecto, o que en su redacción puedan generar cuestionamiento sobre la causación del tributo derivado de estos documentos, no aceptar la oferta mercantil ni sobre el mismo texto ni con un documento posterior, salvo que se acepte mediante la expedición de una orden de compra o venta de bienes o servicios.

\section{Modelo Propuesto para el Segui- miento de Proyectos}

Dada la importancia como se mencionó en un comienzo del proceso de seguimiento de los proyectos con el objetivo de documentar las experiencias adquiridas por sus actores, como aporte con base en el seguimiento y la investigación realizada se considera importante proponer el esbozo de un esquema que sirva como pionero para desarrollar un prototipo en el seguimiento de proyectos.

Adicionalmente, que permita adquirir comparativamente la información de varios proyectos. Con el objetivo de tener una estructura, se plantea una matriz inicial que contiene las diferentes etapas del proyecto y los diferentes aspectos sobre los cuales se recopilará la información en el seguimiento.

\begin{tabular}{|c|l|l|l|l|}
\hline $\begin{array}{c}\text { Aspectos } \\
\text { Etapas }\end{array}$ & Factibilidad & Diseños & Construcción & $\begin{array}{c}\text { Puestaen } \\
\text { Marchay } \\
\text { Mantenimiento }\end{array}$ \\
\hline \multicolumn{1}{|c|}{ Legal } & & & & \\
\hline Administrativo & & & & \\
\hline Financiero & & & & \\
\hline Técnico & & & \\
\hline Comercial & & & & \\
\hline
\end{tabular}

Figura 6. Matriz de organización general de infor- 
mación para seguimiento de proyectos.

Teniendo en cuenta que los aspectos están inmersos en cada una de las etapas de desarrollo de un proyecto, es importante tener en cuenta los procesos llevados a cabo en cada una de éstas.

Por lo general cada uno de los procesos cuenta con un desarrollo conceptual que debe corresponder a un marco teórico, de donde se desprenderá un concepto inicial para desarrollar una actividad.

La actividad desarrollada deberá describirse de manera concreta sin omitir detalles que impidan su objetividad; la ayuda digital juega un papel muy importante, debido a que el apoyo filmico y fotográfico permitirá encausar una idea mucho más completa y detallada sobre los procesos.

Inicialmente, es importante realizar una caracterización del proyecto que permita enmarcarlo bajo una descripción global. Relacionar documentación de proyectos similares es un aporte importante para el seguimiento, puesto que se contaría con material que permitiría establecer comparaciones.

En caso de no contar con documentación de proyectos similares, se iniciaría la documentación del proyecto al cual se le haga seguimiento.

Al realizar el seguimiento es importante desarrollar un análisis comparativo entre el marco conceptual, la documentación de otros proyectos, si existe, el plan inicial del proyecto y el plan ejecutado representado en el material obtenido de la labor. De esta manera se logra establecer las posibles variaciones y sus causas, que permitirán documentar las lecciones aprendidas y recomendaciones a proyectos futuros.

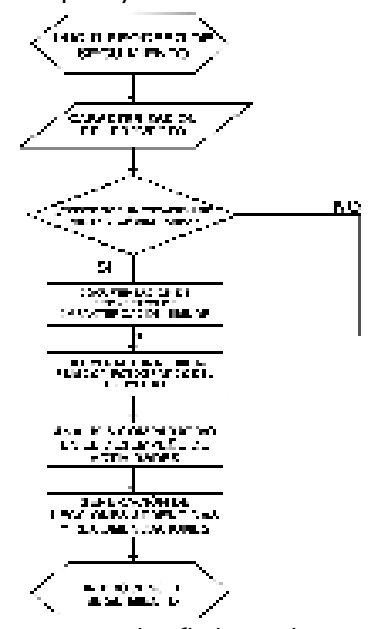

Figura 7. Diagrama de flujo sobre el desarrollo del proceso de seguimiento de un proyecto

\section{Conclusiones}

Al realizar este seguimiento y la investigación vinculada se logró determinar la importancia que tiene para el gerente de proyectos realizar un adecuado seguimiento de los proyectos de manera que arroje lecciones y recomendaciones a tener en cuenta en proyectos futuros y que sirva como apoyo en la toma de decisiones.

Realizar la menor cantidad de cambios al plan inicial de un proyecto es conveniente para reducir el impacto que se puede generar.

Este trabajo permitió establecer una idea preliminar de un modelo de seguimiento para proyectos de construcción.

Una buena gestión por parte de la gerencia de un proyecto es base fundamental en el éxito de éste.

Ante la complejidad que involucra un proyecto de construcción, su manejo puede depender de variables cuyo comportamiento es incierto. Por tal razón, la experiencia que se adquiera no debe permanecer solamente en los actores del proyecto, sino que debe quedar registrada en forma clara y dicho conocimiento ser compartido para no cometer errores a futuro en otros proyectos.

\section{Bibliografía}

Echeverry, D. 2005, Apuntes clase de Gerencia de Proyectos

Sociedad Colombiana de Arquitectos, 1989, Decreto 2090

Wihiler, A. 2004, Seguimiento del desarrollo del proyecto del nuevo edificio Mario Laserna de ingeniería de la universidad de los Andes, Tesis de maestría.

Andreom, D. La seguridad en la construcción de Edificios, Obra sindical "Previsión social", Madrid. Arseg Ltda., 1992, Compendio de normas legales sobre salud ocupacional, artículos de seguridad, Bogotá

Universidad de los Andes, 2003 Bases concurso arquitectónico de anteproyecto del edificio Germania de la Universidad de los Andes; Dirección de Planta Física, Dirección de Planeación y Evaluación, Bogotá 